\title{
Nominata de Avaliadores ad hoc 2010 de Horizonte
}

1. Adriano Correia Silva - UFG

2. Alberto da Silva Moreira - PUC GO

3. Altair Alberto Fávero - UPF

4. Andreia Cristina Lopes Frazão da Silva - UFRJ

5. Antonio José de Almeida Filho - UFRJ

6. Augusto Bach - UNICENTRO

7. Aylton Barbieri Durão - UFSC

8. Breno Hax Junior - UFPR

9. Cassiano Terra Rodrigues - PUC SP

10. Cecilia Cintra Cavaleiro de Macedo - UNIFESP

11. Cicero Cunha Bezerra - UFS

12. Drance Elias da Silva - UNICAP

13. Edebrande Cavalieri - UFES

14. Edin Sued Abumanssur-PUC SP

15. Everaldo Cescon - UCS

16. Faustino Teixeira - UFJF

17. Flávio Augusto Senra Ribeiro - PUC MG

18. Francirosy Campos Barbosa Ferreira - USP

19. Gilbraz de Souza Aragão - UNICAP

20. Haroldo Reimer - PUC GO

21. Hermisten Maia Pereira da Costa - UPM RJ 
22. Ibraim Vitor de Oliveira - PUC MG

23. Jacqueline Wildi Lins - UDESC

24. João Augusto Anchieta Amazonas Mac Dowell - FAJE

25. Jonatas Silva Meneses - UFS

26. José Martins dos Santos Neto - PUC MG

27. José Neivaldo de Souza - FEPAR

28. José Pedro Luchi - UFES

29. Lauro Frederico Barbosa da Silveira - FEESR

30. Lemuel Dourado Guerra Sobrinho - UFPB

31. Leomar Antônio Brustolin - PUC RS

32. Leonildo Silveira Campos - UMESP

33. Lindomar Rocha Mota - PUC MG

34. Luciana Eleonora de Freitas Calado Deplagne - UFPB

35. Magali do Nascimento Cunha - UMESP

36. Magali Oliveira Fernandes - PUC SP

37. Mara Regina do Nascimento - UFU

38. Marcelo Barreira - CAPES/UFES

39. Marcelo Camurça - UFJF

40. Marcelo Fabri - UFSM

41. Marcelo Lapuente Mahl - UFU

42. Márcio Antônio de Paiva - PUC MG

43. Maria Augusta Mundim Vargas - UFS

44. Maria Helena Nery Garcez - IICS-CEU

45. Maria Simone Marinho Nogueira - UEPB

46. Mário Sanches - PUC PR 
47. Marlene Teda Pelzer - FURG

48. Newton Cabral - UCP

49. Orivaldo Pimentel Lopes Junior - UFRN

50. Patrícia Pinna Bernardo - USP

51. Paulo Afonso de Araújo - UFJF

52. Paulo Agostinho Nogueira Baptista - PUC MG

53. Paulo Roberto Margutti Pinto - FAJE

54. Paulo Sérgio Lopes Gonçalves - PUC Campinas

55. Pedro A. Ribeiro de Oliveira - PUC MG

56. Rafael Haddock Lobo - UFRJ

57. Renato Kirchner - USF

58. Roberta Bivar Carneiro Campos - UNIV. SUSSEX, Inglaterra.

59. Rodrigo Ribeiro Alves Neto - UFRN

60. Ronaldo Gomes Alvim - UNICAMP

61. Sandra Jacqueline Stoll - UFPR

62. Sergio Rogerio Junqueira - PUC PR

63. Severino Vicente da Silva - UFPE

64. Silas Guerriero - PUC SP

65. Silvia Regina Alves Fernandes - UFRRJ

66. Simone Fadel - UERJ

67. Susana de Castro Amaral Vieira - UFRJ

68. Valmor da Silva - PUC GO

69. Vitale Joanoni Neto - UFMT

70. Zuleica Dantas Pereira Campos - UNICAP 